\title{
EFECTOS DE LA SOLUCIÓN DE PROBLEMAS SOBRE LOS COMPORTA- MIENTOS DE AUTOCUIDADO DE CUIDADORES DE PACIENTES ONCO- LÓGICOS EN FASE PALIATIVA: UN ESTUDIO PILOTO
}

\author{
EFFECTS OF PROBLEM SOLVING THERAPY ON SELF-CARE BEHAVIORS BY PRIMARY \\ CAREGIVERS OF TERMINAL CANCER PATIENTS: A PILOT STUDY
}

Nancy Elizabeth Rangel-Domínguez ${ }^{1}$, Leticia Ascencio-Huertas ${ }^{2}$, Rosa Elena Ornelas-Mejorada ${ }^{3}$, Silvia Rosa Allende-Pérez ${ }^{2}$, Edgar Landa-Ramírez y Juan José Sánchez-Sosa

1 Universidad Nacional Autónoma de México, México

2 Instituto Nacional de Cancerología, México

${ }^{3}$ Instituto Politécnico Nacional, México

Resumen

Cuidar de un familiar con cáncer avanzado trae consigo una serie de problemas familiares, laborales, económicos, y sociales que impactan negativamente sobre la calidad de vida y la salud física y psicológica de los cuidadores primarios. El objetivo de la presente investigación fue evaluar la eficacia de una intervención basada en la terapia de solución de problemas sobre la adquisición de habilidades de autocuidado, la calidad de vida, y la sintomatología ansiosa y depresiva en cuidadores primarios de pacientes que reciben cuidados paliativos. Se utilizó un diseño de $\mathrm{N}=\mathbf{1}$ de medidas repetidas, con evaluación pre y post- test con los inventarios de ansiedad y depresión de Beck, el inventario de calidad de vida WHOQol Bref y un cuestionario de conductas de autocuidado diseñado ex profeso para esta investigación. Se identificaron mejorías clínicamente significativas en las medidas de sintomatología ansiosa y depresiva, un incremento en la calidad de vida y la identificación de la Terapia de Solución de Problemas como una herramienta útil para afrontar las problemáticas derivadas de las actividades de cuidado. La principal limitante para el desarrollo de la intervención fue que el

\section{Abstract}

Caring for a relative with terminal cancer brings a series of problems in the family, work, financial and social arenas. These problems, in turn, cause a negative impact on the Quality of Life (QOL) of the patients' primary caregivers, including their physical and psychological health. The purpose of the present study was to examine the effects of an intervention based on Problem Solving Therapy on QOL, anxious and depressive symptoms and on the acquisition of self-care skills by terminal cancer patients' primary caregivers. A repeated measures $\mathrm{N}=1$ design was used on pre and post intervention measurements on Beck's anxiety and depression inventories, the WHOQOL-Bref and a questionnaire on self-care expressly designed for the study. Results revealed clinically significant improvements on anxious and depressive symptoms and improvement on the participants' QOL. Problem Solving Therapy resulted a viable and effective approach to cope with the problems posed by caring for a terminal patient and improving self-care skills. A possible drawback of the procedures includes a lengthy intervention

\section{Correspondencia:}

Nancy Elizabeth Rangel Domínguez.

Facultad de Psicología, Universidad Nacional Autónoma de México,

Av. Universidad 3004, Col. Copilco Universidad, Del. Coyoacán,

04510 México, D.F., México.

E-mail: psicnancyrangel@hotmail.com 
tiempo propuesto no correspondió con el promedio de sobrevida de los pacientes atendidos en el servicio.

Palabras clave: Terapia de solución de problemas, cuidadores primarios, ansiedad, depresión, calidad de vida. which was not always commensurate with the survival time of the terminal patients.

Key words: Problem solving therapy, primary caregivers, anxiety, depression, quality of life.

\section{INTRODUCCIÓN}

La familia del paciente con cáncer es afectada por el proceso de enfermedad de forma emocional, cognitiva y conductual $^{(1,2)}$. Estos cambios impactan en mayor medida al cuidador primario informal, es decir, la persona que se hace cargo de brindar los cuidados que otra requiere debido a que se encuentra enferma o tiene alguna discapacidad y por lo tanto, requiere de apoyo para llevar a cabo sus actividades cotidianas, vitales o de relación social $^{(3)}$ generalmente es un familiar, amigo o vecino de la persona enferma.

Actualmente no se cuenta con datos que reflejen cuántos cuidadores primarios existen a nivel mundial, pero, bajo el supuesto de que por cada paciente oncológico existe un cuidador, puede considerarse que en 2008 cerca de 29 millones de personas dedicaron cuidados y atención a enfermos de cáncer a nivel mundial(4). En México, de acuerdo con la Encuesta Nacional de Uso del Tiempo (ENUT), en el 2002 un total de 1.738 .756 personas dedicaban parte del día al cuidado de un familiar enfermo. No se cuenta con los datos que reflejen el tipo de enfermedades que aquejaban a estas personas ${ }^{(5)}$.

Las personas que cuidan de un familiar enfermo experimentan un deterioro importante en su estilo de vida ${ }^{(6)}$ ya que se reducen mucho sus oportunidades de desarroIlo personal y profesional; las relaciones que establecen con otros familiares como los hijos o la pareja se tornan problemáticas $^{(5)}$ y sus recursos económicos pueden ser insuficientes debido a la pérdida de ho- ras laborales o incluso del empleo(7) ${ }^{(7)}$ Los cuidadores presentan también aislamiento social(8) y conducta agresiva hacia el personal de salud ${ }^{(9)}$ y hacia el propio paciente ${ }^{(5)}$.

Se ha encontrado que los cuidadores presentan alteraciones de los patrones de sueño $^{(10)}$ fatiga $^{(11)}$, alteraciones osteomusculares como lumbalgia, cervicalgia y artrosis $^{(12)}$ hipertensión ${ }^{(13)}$, infecciones frecuentes ${ }^{(14)}$; presentan una mayor probabilidad de padecer afecciones cardiacas y alteraciones metabólicas en comparación con los no cuidadores ${ }^{(15)}$ y utilizan sustancias como ansiolíticos y antidepresivos con mayor frecuencia $^{(12)}$. Así, el deterioro que sufre el individuo que cuida de un paciente crónico puede llegar a ser muy serio.

Adicionalmente, se ha documentado que los cuidadores de pacientes oncológicos presentan sintomatología depresiva y ansiosa con mayor frecuencia que la población en general ${ }^{(15-17)}$.

En síntesis, cuidar de un familiar enfermo trae consigo una serie de problemas familiares, laborales, dificultades económicas, sociales así como deterioro de la salud física y psicológica en detrimento serio de la calidad de vida del cuidador ${ }^{(18)}$ en particular cuando brinda cuidado a un familiar con una enfermedad avanzada ${ }^{(19)}$. Estas situaciones pueden llevarlos a problemas de salud graves, convirtiéndose posterior o paralelamente en nuevos pacientes ${ }^{(12)}$.

En este contexto las intervenciones psicosociales dirigidas a mejorar las condiciones de los cuidadores cobran importancia, sin embargo, generalmente estas intervenciones están dirigidas a los pacientes y sus cuidadores, sin considerar que 
las necesidades de ambos son distintas y requieren de diferentes enfoques ${ }^{(20)}$. Pocos trabajos consideran como un punto central el autocuidado de los cuidadores ${ }^{(21)}$ a pesar del notorio impacto que cuidar de un familiar enfermo tiene sobre la salud física y psicológica.

Las intervenciones que han mostrado ser más efectivas han tenido el objetivo de desarrollar las habilidades de afrontamiento de los cuidadores ${ }^{(21)}$, como aquellas basadas en la terapia de solución de problemas, definida como un proceso cognitivoconductual relativamente auto-dirigido, por el cual la persona intenta identificar o descubrir formas efectivas para resolver situaciones problemáticas que enfrenta en el curso de su vida cotidiana. Esta técnica está conformada por cinco elementos que son a un mismo tiempo un conjunto de habilidades y un grupo de procesos: 1 . orientación al problema, 2. definición del problema, 3. generación de soluciones, 4. toma de decisiones y 5. Verificación y puesta en práctica ${ }^{(22,23)}$.

La terapia de solución de problemas ha mostrado ser más eficaz que los tratamientos estándar y tratamientos placebo en la atención de problemas de salud física y psicológica y que no recibir atención psicológica $^{(24)}$. Se ha utilizado en poblaciones de cuidadores primarios de pacientes con diferentes afecciones como múltiples discapacidades $^{(25)}$ y lesiones de la médula espinal $^{(26)}$ en las que mostró ser eficaz en el manejo de la depresión, en el incremento de las habilidades de solución de problemas y la satisfacción con la vida.

Las investigaciones basadas en de la terapia de solución de problemas dirigidas a cuidadores de pacientes con cáncer han reportado que tras la intervención se registran mayores niveles de conocimiento de los recursos de la comunidad y confianza en las habilidades para proporcionar cuidados a su familiar (27) un incremento en las habilidades para solucionar proble- mas y el tipo de estrategias empleadas, así como, el grado de bienestar emocional de los cuidadores, reducción de la tensión emocional, mayor confianza para resolver problemas, un aumento de estrategias de solución consideradas positivas tales como buscar apoyo del equipo médico ${ }^{(28)}$.

Otras investigaciones han reportado que los participantes presentaron una reducción en los niveles de ansiedad, así como, un incremento de las habilidades para solucionar problemas y en la calidad de vida de los cuidadores ${ }^{(29)}$ pero no de los pacientes $^{(30)}$.

La limitación común en estas intervenciones es que están dirigidas a solucionar problemas relacionados con el paciente y no a los problemas que surgen alrededor del cuidador, y específicamente no se dirigen a fomentar el autocuidado, como una medida de prevención para las riesgos de salud generados por las actividades propias del rol que desempeñan y así mejorar su calidad de vida.

Considerando lo anterior se propuso realizar el siguiente estudio el cual tuvo como objetivo evaluar la viabilidad y eficacia de una intervención basada en la terapia de solución de problemas sobre la adquisición de habilidades de autocuidado, la calidad de vida y la sintomatología ansiosa y depresiva de cuidadores primarios de pacientes que reciben cuidados paliativos.

\section{MÉTODO}

\section{Participantes}

Los participantes fueron cuidadores primarios de pacientes atendidos en el Programa de Atención y Cuidados en Oncología del Instituto Nacional de Cancerología (INCan) Se realizó un muestreo no probabilístico de participantes voluntarios ${ }^{(31)}$ y fueron incluidos aquellos que aceptaron participar entre los meses de septiembre y diciembre del 2011. 
Se identificó como cuidador primario aquella persona que ejecutaba las tareas de cuidado que requería un paciente con cáncer avanzado y que no recibía remuneración económica por tal actividad. Se consideraron como criterios de inclusión ser mayor de 18 años, saber leer y escribir, firmar el consentimiento informado y concluir la entrevista general. Como criterios de exclusión se tomó en cuenta que participaran en otro protocolo de investigación o atención psicológica o psiquiátrica, y finalmente el criterio de eliminación fue que el paciente muriera durante el proceso de la intervención y que los cuidadores fueran canalizados a la atención tanatológica brindada por la unidad de cuidados paliativos.
Fueron evaluados trece cuidadores primarios informales cuyas características sociodemográficas se presentan en la tabla 1. De los trece participantes evaluados solo una concluyó la intervención. Las diversas causas por las que los cuidadores salieron de la investigación se enlistan en la tabla 2.

\section{Diseño}

Se empleó un diseño de $\mathrm{N}=1$ de tipo $A B$, donde $A$ da cuenta de las conductas y malestares físicos reportados por los participantes durante la ejecución del plan de solución de problemas y $\mathrm{B}$ durante la verificación del plan.

\section{Tabla 1. Características sociodemográficas de los participantes}

\begin{tabular}{|l|c|}
\hline \multicolumn{1}{|c|}{ Variable } & Frecuencia / Media \\
\hline Sexo & 1 \\
\hline Hombre & 12 \\
\hline Mujeres & \\
\hline Edad & Media: 45 \\
\hline Mínimo 21 \\
Máximo 62 \\
\hline Ocupación \\
\hline Hogar & 9 \\
\hline Empleo fuera del hogar & 4 \\
\hline Parentesco & \\
\hline Cónyuge & 3 \\
\hline Hijo/ hija & 9 \\
\hline Cuidador primario formal & 1 \\
\hline
\end{tabular}

Tabla 2. Causas por las que los participantes salieron del programa de intervención

\begin{tabular}{|l|c|}
\hline \multicolumn{1}{|c|}{ Causa } & Frecuencia \\
\hline Fallecimiento del paciente e ingresó a sesiones de tanatología & 5 \\
\hline Se encontraba en tratamiento psiquiátrico & 1 \\
\hline El paciente ingresó a programa de visita domiciliaria & 2 \\
\hline $\begin{array}{l}\text { El cuidador ya no acudía a consulta en cuidados paliativos por } \\
\text { reasignación de tareas }\end{array}$ & 1 \\
\hline $\begin{array}{l}\text { Se agregaron componentes terapéuticos a la intervención } \\
\text { (Reestructuración cognitiva) }\end{array}$ & 1 \\
\hline El cuidador manifestó no querer continuar en el tratamiento & 1 \\
\hline
\end{tabular}




\section{Variables e instrumentos}

\section{Comportamientos de autocuidado:} Conductas que las personas llevan a cabo con el objetivo de preservar y mejorar su salud a nivel físico y psicológico. Se evaluaron a través de auto-registros conductuales que consideraban la frecuencia con que los cuidadores Ilevaban a cabo tales acciones. Además se empleó un cuestionario de conductas de autocuidado diseñado para esta investigación.

Calidad de vida: Es la percepción que las personas tienen de su posición en la vida considerando el contexto cultural y el sistema de valores en el que se desenvuelve, se consideran además sus metas y expectativas ${ }^{(32)}$ Se midió a través del WHOQol- Bref en su versión adaptada a la población mexicana ${ }^{(33)}$. Considera las dimensiones percepción de salud física, salud psicológica, relaciones sociales y medio ambiente. Tiene una consistencia interna de 0,75.

Ansiedad: Estado de emocional tenso, acompañado de síntomas físicos de activación simpática y procesos cognitivos centrados en la creencia de que la persona se encuentra constantemente en estado de vulnerabilidad ante diversos peligros ${ }^{(34)}$ Para su medición se empleó el Inventario de Ansiedad de Beck, en su versión para población mexicana ${ }^{(35)}$. Esta escala consta de 21 reactivos con respuestas en escala tipo Likert; evalúa la sintomatología física y los pensamientos relacionados con ansiedad. A mayor puntaje obtenido mayor gravedad de la sintomatología. Muestra una consistencia interna de 0,83.

Depresión: Es un grupo de síntomas emocionales, cognitivos, conductuales y somáticos entre los que se encuentran un estado de ánimo abatido o decaído, irritabilidad, pensamientos y sentimientos de culpa, pérdida del interés y/o placer, así como aislamiento social, entre otros $^{(36)} \mathrm{Se}$ valoró a través del Inventario de Depresión de Beck, que ha sido estandarizado para México ${ }^{(37)}$. Está formado de 21 ítems con respuestas en escala tipo de Likert, evalúa la sintomatología depresiva a nivel cognitivo, conductual y emocional. Un mayor puntaje refleja una mayor gravedad de la sintomatología. Su valor de consistencia interna es 0,87 .

Además de los instrumentos mencionados, se empleó una entrevista estructurada diseñada "ex profeso" para esta investigación que recababa información sobre variables como sexo, edad y parentesco con el paciente que permitieron identificar las características generales de los cuidadores. Además exploraba las características del cuidado que los participantes brindaban a sus familiares enfermos, así como las actividades realizadas y el tiempo requerido. Con el objetivo de tener conocimiento de eventos que pudieran afectar la intervención (progresión de la enfermedad, cambio de tratamiento, fallecimiento del paciente) durante cada sesión se realizó un resumen de la misma y se registró en una bitácora clínica.

\section{Procedimiento}

Reclutamiento: Con el objetivo de invitar a los cuidadores a participar de la investigación se entregaron folletos y se colocó un anuncio en el área de recepción del servicio. Aquellos cuidadores que se mostraron interesados en participar recibieron una explicación más extensa sobre la investigación. Quienes aceptaron participar firmaron el consentimiento informado y se acordó cita para inicio del tratamiento.

Evaluación inicial: Se llevó a cabo la entrevista general y la aplicación de los instrumentos para la evaluación inicial. Se inició la evaluación comentando con los cuidadores que la aplicación de los instrumentos tenía como objetivo valorar cuáles eran los problemas más frecuentes que se 
derivaban del cuidado de su paciente, cuáles eran las habilidades con las que contaban y cómo se sentían con la experiencia de cuidar de alguien más. La duración de la evaluación osciló entre 30 y 60 minutos.

Intervención: La intervención se llevó a cabo en 2 sesiones individuales, 1 por semana con una duración aproximada de 90 minutos. El programa estuvo formado por tres etapas:

- Etapa 1. La solución de problemas, su relación con el bienestar emocional y la calidad de vida. En esta fase se brindó psicoeducación a las participantes respecto a la relación entre el bienestar emocional, la calidad de vida y las habilidades de solución de problemas.

- Etapa 2. Solucionando los problemas de cuidadores. En esta etapa el terapeuta leyó las historias de cuidadores primarios contenidas en la guía de solución de problemas y que estaban ya resueltas. A lo largo de la lectura se hizo énfasis en los diferentes pasos de la solución de problemas.

- Etapa 3. Solucionando mis problemas como cuidador. En esta fase se pidió a los participantes que formularan un problema que experimentaban como cuidadores primarios y con ayuda de la terapeuta se implementó y ejecutó un plan de solución de problemas. En la segunda sesión de la intervención se revisaron las metas alcanzadas a través de plan y se realizaron las modificaciones necesarias. Se pidió a las participantes que realizaran el autoregistro de conductas de autocuidado y malestar físico por una semana más y que lo entregaran en la siguiente consulta.

En cada etapa de la intervención se destacó la importancia del autocuidado y la búsqueda de soluciones al respecto. En todas las sesiones se evaluaron los avances de los participantes respecto a la técnica de solución de problemas identificando si era posible dar paso a la siguiente fase de la intervención.
Evaluación final y seguimiento: $\mathrm{Al}$ cubrir las etapas del programa de intervención se aplicaron nuevamente los instrumentos de evaluación y se comentó con los participantes cuáles fueron los cambios que ellos identificaron en sí mismos tras la intervención, su opinión general de la misma y si lo aprendido puede ser generalizado a otros aspectos de su vida.

\section{RESULTADOS}

La participante que concluyó la intervención fue una mujer de 43 años de edad, casada, madre de dos hijos (15 y 17 años), con educación secundaria completa, comerciante. Era la esposa del paciente, a quien cuidaba desde que éste recibió el diagnóstico de cáncer gástrico, 18 meses previos a su ingreso al servicio de cuidados paliativos.

Al momento de la evaluación inicial, la cuidadora dedicaba en promedio cinco horas al día al cuidado del paciente, el resto lo distribuía entre el cuidado de sus hijos y el de su padre quien estaba diagnosticado con cáncer de próstata y recibía tratamiento con quimioterapia. Los cuidados hacia su esposo y su padre consistían en la preparación de alimentos y la administración de medicamentos principalmente. La participante también apoyaba con las labores escolares a sus dos hijos y atendía un puesto de dulces, que era la única fuente de recursos económicos de la familia tras la pérdida del trabajo de su esposo.

Previo a la intervención, la cuidadora presentaba malestares físicos y estaba en protocolo de estudio por una probable pancreatitis, sin embargo tuvo que dejar inconcluso el proceso de atención ante la falta de recursos económicos.

En el mes previo a la evaluación, la cuidadora presentó dolores diversos (lumbalgia y cefalea) casi todos los días y alteraciones del sueño frecuentemente. El prin- 


\section{Tabla 3. Puntajes de ansiedad, depresión y calidad de vida antes y después de la intervención}

\begin{tabular}{|lcc|}
\hline \multicolumn{1}{|c|}{ Variable } & $\begin{array}{c}\text { Puntaje } \\
\text { pre-intervención }\end{array}$ & $\begin{array}{c}\text { Puntaje } \\
\text { post-intervención }\end{array}$ \\
\hline Ansiedad & 23 & 17 \\
Depresión & 24 & 23 \\
Calidad de Vida & 14,67 & 15,85 \\
Salud Física & 14 & 16 \\
Salud Psicológica & 14 & 15 \\
Relaciones sociales & 15 & 16,87 \\
Medio ambiente & 12,50 & 13,14 \\
\hline
\end{tabular}

cipal malestar reportado por la cuidadora fue el cansancio, el que estuvo presente todos los días del mes.

En la evaluación de ansiedad y depresión presentó niveles moderados de ambos grupos de síntomas. La calidad de vida se encontraba disminuida en cada uno de sus dominios. La tabla 3 presenta los puntajes obtenidos por la participante en cada una de las variables.

Respecto a las conductas de autocuidado se encontró que estas eran reducidas. La participante destacó que podía hacer actividades que le resultaban agradables como platicar con sus hijos y padres.

La participante eligió como malestares objetivo el cansancio, pensamientos disruptivos "algo malo pasará", dolor de cabeza y dolor de "estómago". Por otra parte, la conducta de autocuidado que se buscó incrementar fue la toma de descansos a lo largo de la jornada y la reducción de medicamento para el dolor (paracetamol), el cual no había sido indicado por algún médico. Al respecto, previo a la intervención la participante reportó no tomar descansos a lo largo del día y consumir medicamento 3 o 4 veces al día.

Posterior a la intervención se identificó una reducción en la sintomatología ansiosa, más no en la sintomatología depresiva; además se registró un ligero incremento en la calidad de vida en cada uno de los dominios, particularmente en el de salud física. Con relación a las conductas de autocuidado, se registró un incremento en la frecuencia de descansos de la participante lo largo del tiempo de intervención. El registro de la conducta se hizo con base en presencia o ausencia de la misma cada día durante dos semanas. La figura 1 presenta los cambios reportados por la paciente.

Por otra parte, el consumo de medicamento disminuyó rápidamente, ya que al siguiente día de iniciado el plan de solución de problemas la participante reportó no haberlo consumido. Esto se mantuvo a lo largo de las dos semanas entre las sesiones de intervención.

Se encontró también que la participante presentó cambios en la intensidad de la sintomatología física. Los malestares físicos reportados por la participante, con excepción del cansancio, presentaron una tendencia a disminuir durante el periodo en que la cuidadora ejecutó el plan de solución de problemas, sin embargo, una vez que el problema planteado por la participante se resolvió, los puntajes de malestar aumentaron nuevamente, pero sin regresar a los niveles registrados inicialmente. La figura 2 presenta la evolución de la sintomatología física a lo largo de la participación en la intervención. 
Figura 1. Conductas de autocuidado (tomar descansos) antes y después de la intervención

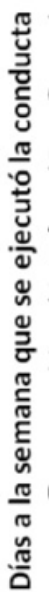

Antes

1a semana

2da semana

Momento de la intervención

Figura 2. Nivel de malestar a lo largo de la implementación del plan de solución de problemas

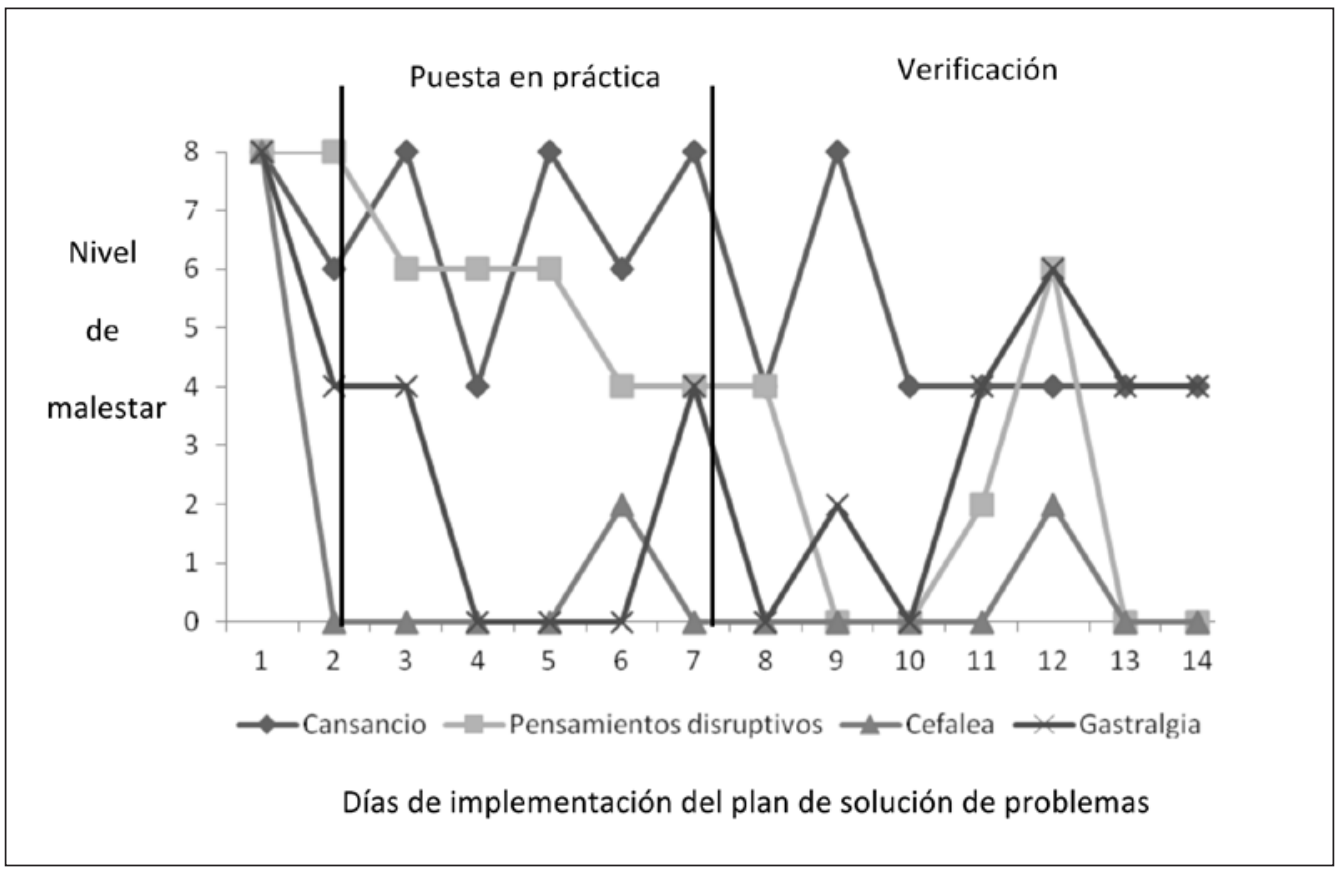




\section{CONCLUSIONES}

Al igual que en investigaciones previas se encontró que, es viable utilizar la terapia de solución de problemas en la población de cuidadores primarios de pacientes con cáncer y que ésta tiene un impacto positivo sobre la calidad de vida y la reducción de la ansiedad ${ }^{(29)}$. Sin embargo, con respecto a la sintomatología depresiva se encontró que ésta no disminuyó, lo cual es contrario a lo reportado por otras investigaciones, en las que, la intervención estuvo dirigida a cuidadores de personas con múltiples discapacidades y lesiones en médula espinal ${ }^{(25,26)}$; esto puede deberse a las diferencias que existen en la dinámica de atención a los diferentes tipos de patologías y al mal pronóstico a corto plazo con el que cuentan las personas con cáncer avanzado en cuidados paliativos. Las cuidadoras de éste tipo de pacientes desempeñan su rol acompañadas de la inminente muerte de su familiar, lo que puede favorecer la presencia de otras variables de orden psicológico como el proceso de duelo anticipado. Dado que no era el objetivo del presente estudio la variable duelo no fue abordada sin embargo se considera importante incluirla en otras investigaciones que se realicen con cuidadores de pacientes en cuidados paliativos.

Por otra parte, es importante destacar el papel central que en esta investigación se dio a la variable conductas de autocuidado, ya que de acuerdo con la literatura previa, ésta no había sido abordada ${ }^{(21)}$. En el caso de la participante que concluyó la intervención se registró un incremento en la frecuencia de conductas de autocuidado, lo cual cobra importancia al considerar que ha sido documentado ampliamente un deterioro en la salud física y psicológica de las cuidadoras primarias, la que las coloca en una situación de alta fragilidad. El que la intervención basada en la terapia de solución de problemas contribuya en la ejecución de conductas de autocuidado abre la puerta a la posibilidad de contar con intervenciones dirigidas a la prevención y atención de problemas de salud en una población altamente vulnerable.

A lo largo de la investigación se presentaron una serie de dificultades para la ejecución de la intervención que no habían sido reportados previamente, como la expectativa de vida de los pacientes al momento de ingresar a cuidados paliativos. En el caso de la unidad donde se llevó a cabo la investigación el promedio de sobrevida de los pacientes es de 28 días, durante los cuales presentan, con frecuencia, un deterioro progresivo que demanda del cuidador más atenciones e implica una mayor dependencia, por lo que las oportunidades de las cuidadoras para acudir a las sesiones del taller se redujeron significativamente. Otras variables que impidieron que las cuidadoras se presentaran a todas las sesiones programadas fueron: el alto porcentaje de participantes originarios de lugares distintos al Distrito Federal que decidieron regresar a sus lugares de origen cuando el paciente presentó un mayor deterioro, con lo que se interrumpió la atención al cuidador primario dentro de la unidad. El factor económico también impactó sobre la adhesión al tratamiento ya que en algunos casos las cuidadoras no acudieron a las sesiones debido a que implicaba un gasto económico, sobre todo si acudían de otro estado y por lo tanto pedían el apoyo de familiares o amigos que habitaban más cerca del instituto para acudir a las consultas por lo que el acceso a la población objetivo se limitó.

Ante estos hallazgos se propone continuar con la investigación respecto al impacto de intervenciones dirigidas a cuidadores de pacientes con cáncer avanzado, que estén basadas en la terapia de solución de problemas y se desarrollen en pocas sesiones. Se sugiere además considerar variables como el duelo anticipado y el 
nivel de funcionalidad y dependencia del paciente al momento del ingreso a cuidados paliativos, para identificar los alcances de la terapia de solución de problemas. Es importante también considerar que el diseño empleado en la presente investigación no consideró mecanismos de control experimental debido a las dificultades para que los participantes continuaran en la intervención, ante esto cabe la sugerencia de utilizar otras estrategias como la incorporación de un grupo control, con el objetivo de incrementar tanto la validez interna como externa de la investigación.

A pesar de las múltiples dificultades para la realización de la intervención se encontró que las cuidadoras identificaban a la terapia de solución de problemas, en particular al paso de formulación de soluciones, como una herramienta valiosa para la resolución de problemáticas psicosociales, de la misma forma lo reportado por otros autores. Entre las principales aportaciones de la investigación se encuentra que estuvo diseñada para atender a las necesidades de las cuidadoras respecto a las problemáticas que enfrentan al cuidar de un familiar enfermo, a diferencia de otras investigaciones que estaban centradas en el desarrollo de habilidades para el mejor cuidado de los pacientes.

También se destaca que en la investigación se guio hacia la mejora de estrategias de autocuidado de los cuidadores, lo que no había figurado como un objetivo central de investigaciones previas. Si bien en la presente investigación no se logró obtener datos con significancia estadística si se identificaron cambios clínicos en las habilidades de las cuidadoras para llevar a cabo conductas de autocuidado que permitieron a su vez mejoras en otras áreas como la social y la relación con la familia.

Con base en los hallazgos de esta investigación se proponen algunas ideas generales en pro de un mejor desempeño y probablemente, resultados más eficaces:
1) Fomentar y ejecutar la difusión de los cuidados paliativos con el objetivo de que los pacientes y sus cuidadores sean referidos de manera temprana y de esta forma la diada se beneficie de la atención especializada. En el caso de los cuidadores esto permitirá que tengan un mayor tiempo para la adaptación, adquisición y desarroIlo de habilidades tales como la solución de problemas, 2) Se propone que en siguientes intervenciones se tomen en consideración los recursos como los servicios de call center para hacer más cercana la intervención en solución de problemas a aquellos cuidadores que tienen que alejarse físicamente del servicio. Se puede utilizar estos recursos en el diseño de nuevas intervenciones que sean cortas pero brinden un mayor seguimiento del desempeño de las habilidades de las cuidadores.

AGRADECIMIENTOS: El presente artículo forma parte del trabajo de investigación de la primera autora para la obtención del grado de Maestría en Psicología bajo la supervisión del último autor. Para la realización de la investigación se contó con apoyo del Consejo Nacional de Ciencia y Tecnología (CONACYT).

\section{REFERENCIAS BIBLIOGRÁFICAS}

1. Baider L. Cáncer y familia: aspectos teóricos y terapéuticos. RIPCS/IJCHP 2003;3505-20.

2. Mesquita E, Magalhaes da Silva R, De Almeida AM, Carvalho AF, Mota CD. Comportamiento de la familia frente al diagnóstico de cáncer de mama. Enfermería Global 2007;6:1 -10.

3. García M, Mateo, R, Gutiérrez, P. Cuidados y cuidadores en el sistema Informal de salud. Investigación Cuantitativa 1999 Àcceso 13 de septiembre de 2013]. Disponible en: http://www.easp.es/publicaciones/descargas/EASP_Cuidados $\% 20$ y\%20cuidadores $\% 20$ en $\% 20$ sistema $\% 20$ 
informal\%20de\%20salud\%20Investigacion\%20cuantitativa.pdf.

4. Bray F, Ren J, Masuyer E, Ferlay J. Global estimates of cáncer prevalence for 27 sites in the adult population in 2008. Int J Cancer 2012;132:1133-45. Doi: 10.1002/ijc.27711

5. Nigenda G, López-Ortega M, Matarazzo C, Juárez-Ramírez C. La atención de los enfermos y discapacitados en el hogar. Retos para el sistema de salud mexicano. Salud Publica Mex 2007;49:286-94

6. Cameron JI, Franche R L, Cheung A M, Stewart D E. Lifestyle interference and emotional distress in family caregivers of advanced cancer patients. Cancer 2002;94:521-7. Doi: 10.1002/cncr.1012

7. Sherwood P R, Donovan H S, Given C W, Lu X, Given B A, Hricik A, Bradley S. Predictors of employment and lost hours from work in cancer caregivers. Psychooncology 2008;17:6: 598-605. Doi: 10.1002/ pon. 1287

8. Stefani D, Seidmann S, Pano C, Acrich L. Los cuidadores familiares de enfermos crónicos: sentimiento de soledad, aislamiento social y estilos de afrontamiento. Rev Latinoam Psicol 2003;35:55-65.

9. Moreno M, Mateos M. Agresividad de familiares cuidadores de enfermos oncológicos hospitalizados. Psicooncología 2004;1:127-38.

10. Wei-Chung EW, Tsai YY, Chang TW, Tsao CJ. Quality of sleep and quality of life in caregivers of breast cancer patient. Psychooncology 2007;16:950-5.Doi: 10.1002/ pon. 1167

11. Swore BA, Schumacher KL, Dodd M, Paul SM, Cooper B, Lee Ket al. Trajectories of fatigue in family caregivers of patients undergoing radiation therapy for prostate cancer. Res Nurs Health 2009;32:125-39. Doi: 10.1002/nur.20312 49

12. Seira MP, Aller A, Calvo A. Morbilidad sentida y diagnosticada en cuidadores de pacientes inmovilizados de una zona de salud rural. Rev Esp Salud Pública 2002;76:713-21
13. Kim Y, Spillers R. Quality of life of family caregivers at 2 years after a relative's cancer diagnosis. Psychooncology 2010;19:43140. Doi: 10.1002/pon.1576

14. Floriani C, Roland F. Cuidador do idoso com câncer avânçado: um ator vulnerado. Cad. Saúde Pública 2006;527-34. Doi: 10.1590/S0102-311X2006000300007

15. Rivera HR. Depression symptoms in cancer caregivers. Clin J Oncol Nurs 2009;13:195-202. Doi:10.1188/09. CJON.195-202

16. Carol M. Grandmothers who are caregivers are more prone to stress and depressive symptoms. New York: Medical Study News 2001;123-32.

17. Grov EK., Dahl A, Moum T, Fossa D. Anxiety, depression, and quality of life in caregivers of patients with cancer in late palliative phase. Ann Oncol 2005;16:1185-91. Doi: 10.1093/annonc/ mdi210

18. Neugaard B, Andresen E, McKune S L, Jamoom E W. Health-related quality of life a National Sample of caregivers: Findings from the behavioral risk factor Surveillance system. J Happiness Stud 2008;9:559-75. Doi: 10.1007/s10902-008-9089-2

19. Tang S, Li C, Chen C. Trajectory and determinants of the quality of life of family caregivers of terminally ill cancer patients in Taiwan. Qual Life Res 2008;17:387-95. Doi: 10.1007/s11136-008-9316-7

20. Ussher J M, Pertz J, Hawkins Y, Brack M. Evaluating the efficacy of psycho-social interventions for informal carers of cancer patients: A systematic review of the research literature. Health Psychol Rev 2009;3:85107. Doi: 10.1080/174319093033401

21. Northouse L L, Katapodi M, Song L, Zhang L, Mood DW. Interventions with family caregivers of cancer patients. Metaanalysis of randomized trial. CA Cancer J Clin 2010;60, 317-339. Doi: 10.3322/ caac. 20081.

22. Poinsot R, Antoine P. Une aproche psychothérapique méconnue: la résolution 
de problemas sociaux. Annales MédicoPsychologiques 2007;165:638-44. Doi: 10.1016/j.amp.2006.05.017

23. Nezu A. Terapia de Solución de Problemas para pacientes con cáncer. Rev Mex Psicol 2001;18:185-92.

24. Malouff JM, Thorsteinsson E B, Schutte NS. The efficacy of problem solving therapy in reducing mental and physical problems: $\mathrm{A}$ meta-analysis. Clin Psychol Rev 2007;27:4657. Doi: 10.1016/j.cpr.2005.12.005

25. Elliott TR, Berry J W, Grant J S. Problemsolving training for family caregivers of women with disabilities: A randomized clinical trial. Behav Res Ther 2009;47:54858. Doi: 10.1016/j.brat.2009.03.006 46

26. Elliott TR, Brossart D, Berry JW, Fine PR. Problem-solving training via videoconferencing for family caregivers of persons with spinal cord injuries: A randomized controlled trial. Behav Res Ther 2009;46:1220-9. Doi: 10.1016/j.brat.2008.08.004

27. Bucher J A, Loscalzo M, Zabora J, Houts P, Hooker C, BrintzenhofeSzoc K. Problem-solving cancer care education for patients and caregivers. Cancer Prac 2001;9:66-70. Doi: 10.1046/j15235394.2001.00900266.x

28. Cameron JI, Shin JL, Williams D, Stewart D E. A brief problem-solving intervention for family caregivers to individuals with advanced cancer. J Psychosom Res 2004; 57:137-43. Doi: 10.1016/S00223999(03)00609-3

29. Demiris G, Parker D, Washington K, Thomas L, Fruehling M, Haggarty-Robbins et al. A problem solving intervention for hospice caregivers: A pilot study. J Palliat Med 2010;13:1005-11. Doi:10.1089jpm.2010.0022
30. Meyers FJ, Carducci M, Loscalzo MJ, Linder J, Greasby T, Beckett L. Effects a problem-solving intervention (COPE) on quality of life for patients with advanced cancer on clinical trials and their caregivers. Simultaneous care educational intervention (SCEI): linking palliation and clinical trials. J Palliat Med 2011;14:465-73. Doi: 10.1089/jpm.2010.0416.

31. Hernández R, Fernández C, Baptista, P. Metodología de la investigación. [Research Methodology] (4ta ed.). México: McGraw-Hill 2006

32. Organización Mundial de la Salud [OMS] (1998). Promoción de la salud. Glosario. Disponible en http://www.bvs.org.ar/pdf/ glosario_sp.pdf

33. Sánchez-Sosa JJ, González-Celis R. Evaluación de la calidad de vida desde la perspectiva psicológica. En: Caballo V., coordinador. Manual para la evaluación clínica de los trastornos psicológicos: Trastornos de la edad adulta e informes psicológicos. Madrid: Editorial Pirámide 2006.p.473-92

34. Freeman A, DiTomasso R. Conceptos cognitivos de la ansiedad. En: Stein, D, Hollander, E, editores. Tratado de los trastornos de ansiedad. Barcelona: Ars Medica, 2004.p. 85-95.

35. Robles R, Varela R, Jurado S, Páez F. Versión mexicana del Inventario de Ansiedad de Beck: propiedades psicométricas. Rev Mex Psicol 2008;8:211-7.

36. Alberdi J, Taboada O, Castro C, Vázquez C. Depresión. Guías clínicas 2006;6:1-6.

37. Jurado S, Villegas E, Méndez L, Rodríguez F, Loperena V, Varela R. La estandarización del Inventario de Depresión de Beck para los residentes de la ciudad de México. Salud Mental 1998;21:26-31. 